$R M x A C, \mathbf{5 3}, 158-160(2021)$

(C) 2021: Instituto de Astronomía, Universidad Nacional Autónoma de México

https://doi.org/10.22201/ia.14052059p.2021.53.32

\title{
ORBITAL UNCERTAINTY ESTIMATION SUPPORT FOR AUTONOMOUS SPACE DEBRIS OBSERVATION
}

\author{
H. Jiang ${ }^{1}$, J. Liu ${ }^{1,2}$, and H. W. Cheng ${ }^{1,2}$
}

\begin{abstract}
RESUMEN
El aumento continuo de los desechos espaciales ha supuesto grandes riesgos de impactos para los sistemas espaciales existentes y los vuelos espaciales tripulados. El conocimiento exacto de los errores de propagación de las órbitas de los desechos espaciales es esencial para muchos tipos de usos, como la tarea de red de vigilancia espacial, los análisis de conjunción, etc. Desafortunadamente, el error de propagación no está disponible para un elemento de dos líneas (TLE). En este documento se propone un nuevo método para estimar la incertidumbre de TLE basado en el modelo de redes neuronales. Las propiedades del objeto, el entorno espacial y el intervalo de tiempo previsto se consideran como los datos iniciales para la red, mientras que los errores de propagación en la dirección descendente, normal y conormal son los resultados a obtener. Para asegurar que la orbita elegida para el entrenamiento no es estable, solo se utilizan escombros y cuerpos de cohetes. Se demuestra la eficiencia de la red con algunos objetos con datos TLE continuos. En general, el método demuestra ser preciso, computacionalmente rápido y robusto, y es aplicable a cualquier objeto en el catálogo de satélites, especialmente para aquellos objetos recién lanzados.
\end{abstract}

\section{ABSTRACT}

The continually increased space debris have posed great impact risks to existing space systems and human space flight. Accurate knowledge of propagation errors of space debris orbit is essential for many types of uses, such as space surveillance network tasking, conjunction analysis etc. Unfortunately, propagation error is not available for a two-line element (TLE). In this paper, a new TLE uncertainty estimation method based on neural network model is proposed. Object properties, space environment and predicted time-span are considered as the input of the network, the propagation errors in the direction of downrange, normal and conormal are as the output of the network. In order to assure the chosen orbit for training is not stable, only debris and rocket bodies are used. The network's efficiency is demonstrated with some objects with continuous TLE data. Overall, the method proves accurate, computationally fast, and robust, and is applicable to any object in the satellite catalogue, especially for those newly launched objects.

Key Words: methods: miscellaneous — Space vehicles — techniques: miscellaneous

\section{INTRODUCTION}

A growing number of space activities have created an orbital debris environment that poses increasing impact risks to existing space systems and human space flight (Klinkrad 2010). In order to protect the on-orbit space system, accurate orbital elements of space debris are needed, so does the need of associated covariance to improve the knowledge of orbital propagation. The covariance describing the accuracy of a space debris orbital element is an important input for many scenarios, such as conjunction analysis and re-entry predictions, which are increasingly important for operating in today's space

\footnotetext{
${ }^{1}$ National Astronomical Observatories, Chinese Academy of Sciences, 20A Datun Road, Chaoyang District, Beijing, P.R.China 100101. (jhai@nao.cas.cn).

${ }^{2}$ University of Chinese Academy of Sciences, No.19(A) Yuquan Road, Shijingshan District, Beijing, P.R.China 100049 .
}

environment. Through propagation, the probability of potential collisions and a spread of impact locations and times anticipated can be calculated, these efforts help significantly in managing and mitigating the hazards of space debris.

Two-line elements (TLEs) present the most comprehensive and up-to-date source of man-made space objects and are widely used in many activities. Despite the importance of TLEs, they have many drawbacks, such as, low accuracy, miss maneuvers, and perhaps most importantly, lack of uncertainty information. The lack of uncertainty information of TLEs has initiated numerous studies. A wide range of studies (Yim et al. 2012; Kahr et al. 2013; Geul et al. 2017) has been conducted to derive the uncertainty information. These approaches differ greatly in complexity, accuracy and applicability. Most of the existing methods relying on external data have 
many limitations, such as data are not available for the far majority of objects. Moreover, uncertainties derived for a few objects are hard to extrapolate across the population or time due to their dependency on object properties (size, shape, etc.), orbital elements (semi-major axis, eccentricity, inclination, etc.), variability of the environment (solar radio flux, etc.), and the models and determination routines of TLEs.

In this paper, a new orbital uncertainty estimation method based on neural network model is proposed. A multi-layer perception neural network with two-layer of neurons is used in this work. For each set of input data, the network provides a set of orbital uncertainties, which corresponds to a nonlinear function. Since the problem under investigation is a nonlinear process, the activation function applied to the hidden-neuron is the hyperbolic tangent sigmoid function. For the output layer, a linear function was considered. The inputs are object properties, orbital elements, space environment parameters and prediction time-span. The network's efficiency is also validated with real TLE data. From the experiments, the method proves accurate, computationally fast, and robust, and is applicable to any object in the satellite catalogue, especially for those newly launched objects.

\section{NEURAL NETWORK MODEL}

An artificial neural network (NN) (Haykin 1999) is a parallel distributed system consisting of massively interconnected simple processing units, also referred to as artificial neurons. It is a type of nonlinear model representation inspired by biological neural networks.

In the neuron model, signal $x_{i}$ at the input of the synapse $i$ connected to neuron $j$ is multiplied by the synaptic weight $\omega_{j i}$. This network is, therefore, trained by an iterative adjustment of the synaptic weights using both known input and output data. This kind of network have the ability to learn and generalize, that is, they are able to provide reasonable outputs for inputs not used during the training process. They are composed by interconnected layers of neurons, in which the output $y_{j}$ of a single neuron $j$ with $m$ inputs is given by the nonlinear weighted sum

$$
y_{j}=\phi\left(\sum_{i=1}^{m} \omega_{j i} x_{i}+b_{j}\right)
$$

where $b_{j}$ is the bias, $x_{1}, x_{2}, \cdots, x_{m}$ are the input signals, $\omega_{j 1}, \omega_{j 2}, \cdots, \omega_{j m}$ are the synaptic weights

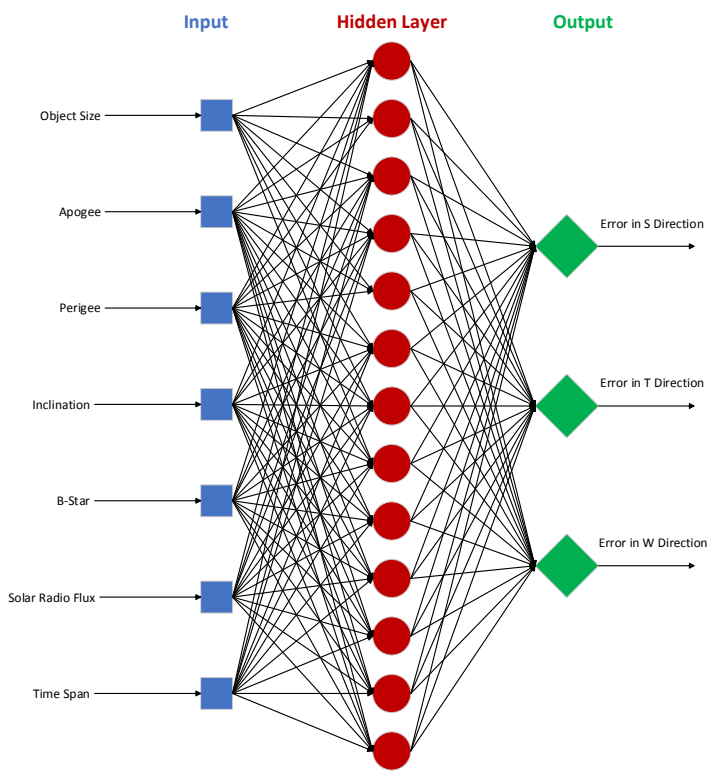

Fig. 1. Schematic representation of the improved NN model.

of neuron $j ; \phi$ is the activation function and $y_{j}$ is the output signal of neuron $j$.

Since the NN is composed of different layers of neurons, the output of a single neuron, as given in Eq. 1, is connected to the input of another neuron. In this case, the output of a NN with a single node in the output layer and a single hidden layer is a nonlinear function with the following structure

$$
y_{o}(k)=\phi_{o}\left\{b_{o}+\sum_{j=1}^{N_{j}} \omega_{j}^{o} \phi_{j}\left(b_{j}+\sum_{i=1}^{N_{i}} \omega_{j i}^{h} x_{i}(k)\right)\right\}
$$

where $y_{o}(k)$ is the output of the $\mathrm{NN}$ at instant $k$; $x_{i}$ is the $i$-th input, $\omega_{j i}^{h}$ indicates a weight of the hidden layer that connects the $i$-th input (which is the $i$-th output of the previously layer) to the $j$-th neuron of the hidden layer. $N_{i}$ is the number of input signals and is the number of neurons in the hidden layer. The biases and the activation functions are represented by $b$ and $u$, respectively. Finally, the variables indicated by an ' $\mathrm{o}$ ' are related to the output neuron (Aguirre et al. 2004). The parameters of the proposed multi-layer NN are estimated using the back propagation algorithm (Haykin 1999).

\section{PROPOSED NETWORK AND TRAINING}

An illustrative scheme of the NN model proposed in this work is shown in Figure 1, which is an improved version of that was proposed in (Jiang e al. 2018). The input layer consists of 7 artificial neurons, the hidden layer consisting of 13 artificial neurons and the output layer has 3 artificial neurons. 
The number of neurons in each layer is fixed. For each set of input data (are object Size, apogee, perigee, inclination, B-Star, solar radio flux, and time-span, the network provides a propagation errors in $S, T$ or $W$ direction, which corresponds to $y_{o}(k)$ described in Eq. 2. Since the problem under investigation is a nonlinear process, the activation function applied to the hidden neurons is the hyperbolic tangent sigmoid function presented in Eq. 3.

$$
\Phi(n)=\frac{2}{1+e^{-2 n}}-1
$$

For the output layer, a linear function was considered.

\subsection{Inputs}

Since TLE propagation errors are influenced by object size, orbital parameters, predicted time-span and the solar activity, the input parameters of the NN model are detailed as follows:

Object Size can be calculated with SEM model, the RCS data of each object are selected from the satellite situational report provided by Space-Track website.

Orbital parameters are directly influence the propagation errors, especially the semi-major axis, eccentricity, inclination and B-Star. Orbital data used in this work were obtained from space-track website.

Prediction time-span is another important factor that influences TLE propagation errors. In order to account predicted time-span variability, a series of TLE data are considered in this work.

The $10.7 \mathrm{~cm}$ solar radio flux (F 10:7) is one of the most used indexes to interpret solar activity. Solar radio flux data used in this work were obtained from NOAA database, available online.

\subsection{Training}

In order to evaluate the performance of the NN model, the TLE data of all objects from September 1, 2019 to September 30, 2019 are investigated. For each object, the TLE data in the first 27 days are selected to provide training data to the $\mathrm{NN}$ network and the data in the last 3 days are used to test the network and verify its performance.

Known input and output data are required to train the network. In training process, for a known input vector, the NN provides an estimated output. This output is then compared with the expected output value, and the error for each training pattern is sent back to the hidden layers by the Backpropagation algorithm, updating the NN weights. In

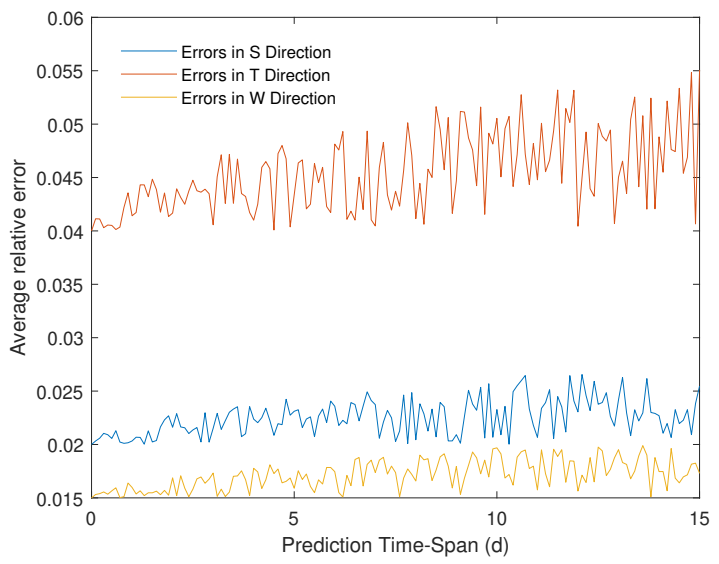

Fig. 2. Estimated STW average relative errors of ATLAS 2A CENTAUR R/B within 15 days.

order to avoid overfitting or overtraining, $90 \%$ of the training set was used to the training procedure and $10 \%$ was used to validate the model.

\section{EXPERIMENTAL RESULTS}

The performance of the new NN model is evaluated with the average relative error, which is calculated according to Eq. 4.

$$
\epsilon=\frac{\left|N_{e r r}-C_{e r r}\right|}{C_{e r r}} \times 100 \%
$$

where $N_{\text {err }}$ is NN estimated error and $C_{\text {err }}$ is calibrated error.

Figure 2 shows the performance of the new model fitted well with the prediction data within a 15-day prediction time-span, which verified the validation of the proposed model in the work.

\section{CONCLUSION}

A new orbital uncertainty estimation model is proposed based on neural network model; experiment results demonstrated the new model can provide good estimations of short-term TLE uncertainty for space objects. It is worth mentioning that many characterization of space debris does not considered in the proposed NN model, more work should be done in the future research.

\section{REFERENCES}

Aguirre, E., et al. 2004, PhRv, 69, 1

Geul, J., et al. 2017, AdSpR, 59, 10

Haykin, S. 1999, Neural Networks: A Comprehensive Foundation, 2nd., Pearson Education, Delhi, India. Kahr, E., et al. 2013, JSpRo, 50, 433

Klinkrad, H. 2010, Space Debris: Models and Risk Analysis, John Wiley \& Sons, Ltd.

Yim, H. \& Chung, D. 2012, JASS, 29, 3

Jiang, H., et al. 2018, AMOS 